\title{
Erratum to: Spectral features of conjugated bichromophores: porphyrins with curcumin
}

\author{
Aleksandr Starukhin ${ }^{1, \star}$, Aleksander Gorski², Tatsiana Pavich ${ }^{1}$, and Valerii Kniukshto ${ }^{1}$ \\ ${ }^{1}$ B.I. Stepanov Institute of Physics, National Academy of Sciences of Belarus, Minsk 220072, Belarus \\ ${ }^{2}$ Institute of Physical Chemistry, Polish Academy of Sciences, Warsaw 01-224, Poland
}

Original article: EPJ Web of Conferences 190, 04023 (2018), https://doi.org/10.1051/epjconf/201819004023

The acknowledgement of the article:

“This work was supported by European Union’s Horizon 2020 research and innovation program, grant agreement № 645628 and the Belarusian-Polish project № F18PLSHG-003.”

should be replaced by the following text:

This project has received funding from the European Union's Horizon 2020 research and innovation programme under the Marie Skłodowska-Curie grant agreement No. 645628, the Polish Ministry of Science and Higher Education grant W77/H2020/2017 and the Belarusian-Polish project № F18PLSHG-003. 